\title{
Variables psicológicas y seropositividad al VIH: aplicación de la medicina conductual en el análisis empírico de una interrelación
}

\author{
Roca, X. ${ }^{1}$, Tejero, A. ${ }^{\prime}$, Trujols, J. ${ }^{1}$, Soler, Q. ${ }^{1}$, Cid, J. ${ }^{1}$, Campins, M.J. ${ }^{1}$, Pinet, C. ${ }^{2}$ \\ 1 Psicólogo Clínico. 2 Psiquiatra. \\ Unitat de Conductes Addictives. Programa SANT PAU-CITRAN. Departament de Psiquiatria. Hospital de la Santa Creu i Sant Pau.
}

Enviar correspondencia a:

Antonio Tejero. Unitat de Conductes Addictives. Servei de Psiquiatria. Hospital de la Santa creu i Sant Pau.

Avda. Sant Antoni Ma Claret 167. 08025 Barcelona. Telf: 932919131

\section{Resumen}

El objetivo de este estudio es el de analizar la relación que existe entre diversas variables psicológicas como la alexitimia, el estrés y el coping, medidas a través de distintos cuestionarios y la infección o el posible riesgo de infección de VIH, en una muestra de 107 pacientes adictos a opiáceos. Se realizó un análisis factorial mediante rotación varimax con las distintas escalas obteniéndose una solución factorial de tres factores que explicaba el $48.8 \%$ de la varianza. Los tres factores obtenidos son: Factor 1: Desesperanza, Factor 2: Racionabilidad y Emocionabilidad, Factor 3: Acción. Se comentan las posibles estrategias terapéuticas teniendo en cuenta estos factores.

Palabras clave: Adictos a opiáceos, VIH, estrés, coping, alexitimia.

\section{Summary}

The objective of this study is to analize the relationship between different psichological variables like alexithymia, stress and coping assessed by self-reports and the infection or the possible infection of HIV, in a sample of 107 opiate addicts patients. We realized a factorial analysis with the different selfreports and we obtained a factorial solution of three factors with $48.8 \%$ of explained variance. This factors were: Factor 1 : Helplessness, Factor 2: Rationability and Emotionability, Factor 3: Action. Different therapeutic strategies bearing in mind these factors are discussed.

Key words: Opiate addicts, HIV, stress, coping, alexithymia.

(1962, 1986) - que han surgido como alternativas científicas al modelo biomédico.

Considerando, pues, las implicaciones de estos modelos, el hecho de poder delimitar las relaciones entre distintas variables psicológicas relevantes en cualquier enfermedad crónica y cómo se relacionan con otras variables (médicas y sociales) es uno de los objetivos básicos para la consolidación de estos modelos y ayudarían a determinar qué intervenciones terapeúticas son las más adecuadas y eficaces para conseguir una mayor implicación del paciente en el tratamiento, mayor cumplimiento de normas terapéuticas y una mejor calidad de vida.

La Psiconeuroinmunología (Ader, 1981; Ader y cols.; 1991) ha sido uno de los modelos que más ha estudiado la relación entre la enfermedad y diversas variables psicológicas. Desde este modelo autores como Solomon y Temoshock (1990) ya plantearon algunas questiones fundamentales para el estudio de 
la relación entre la infección del VIH y variables psicológicas. Desde estos modelos se ha estudiado las variaciones de la función inmune en relación con variables psicológicas como el estrés, el coping y rasgos de personalidad, entre otras.

El estrés es una de las variables que se ha asociado clásicamente a enfermedades físicas y/o psicológicas, habiéndose demostrado su capacidad inmunosupresora en diversos estudios tanto en animales como en seres humanos (Solomon, 1981), hecho que determina su importancia en todo proceso de enfermar e implica necesariamente la evaluación de dicha variable y la implementación de programas de intervención para incidir sobre dicha variable en personas que sufren enfermedades crónicas. Uno de los métodos tradicionales para evaluar el estrés ha sido mediante escalas de acontecimientos vitales que adolecen, sin embargo, de no valorar adecuadamente el estrés percibido por el sujeto, cuestión de gran importancia si tenemos en cuenta la concepcion de estrés y estrategias de afrontamiento de Lazarus y Folkman (1984). Factores amortiguadores de estrés, como el locus de control, el apoyo social y las estrategias de afrontamiento, deben evaluarse porque pueden facilitar la intervención terapéutica a fin de disminuir el grado de estrés percibido. La importancia de estas variables en la evolución de las enfermedades ha sido descrita en diferentes estudios. En este sentido, Watson y col. (1988) en un estudio de seguimiento de pacientes mastectomizadas, hallaron que la variable que mejor predecía la supervivencia de la paciente al cabo de 10 años era el tipo de adaptación ("Espíritu de Lucha") a la enfermedad que desarrollado por la paciente, evaluada mediante la Escala de Ajuste Mental al Cáncer (EAM) tres meses después de la mastectomía para evitar medir la primera reaccción emocional. Estudios recientes (Byrnes et al., 1998) realizados en una muestra de mujeres seropositivas al VIH y al papilovirus han encontrado que una actitud negativa puede ser asociada a bajos rendimientos inmunológicos lo que supone una mayor actividad de este virus y un incremento del riesgo de manifestar una displasia cervical.

Por otra parte, el locus de control, atribución que hace el sujeto respecto a si el control de una situación - como la enfermedad -depende de él (interno) o de los demás (externo), influye en la percepción de los acontecimientos de tal manera que sujetos con un control externo se sienten más indefensos y estresados ante situaciones susceptibles de generar respuestas de estrés que los que presentan un locus de control interno (Kelly y cols., 1989; Kelly y cols., 1990). El locus de control interno respecto al curso de la enfermedad ha sido asociado a altas puntuaciones en la subescala "Espíritu de Lucha" del EAM, no existiendo influencia del estado anímico de los pacientes sobre estas variables mientras que sí existía una correlación positiva entre la escala "Ansiedad-Preocupación" del EAM - que representa una respuesta patológica a la situación de padecer cáncer - y la atribución interna respecto a la causa del cáncer (Watson y col., 1988). El Apoyo Social ha sido destacado por diferentes autores como uno de los elementos más importantes en el proceso de enfermar por la facilidad de intervención sobre dicha variable y su significativa influencia en la modulación de aspectos emocionales y de afrontamiento ante situaciones estresantes (Zich y Temoshock, 1990). En un estudio longitudinal realizado en una muestra de hombres homosexuales y bisexuales seropositivos al VIH se ha observado que los sujetos que mostraban un menor apoyo social manifestaban una disminución significativa de los niveles de los linfocitos CD4 (Miller et al., 1997).

Determinados rasgos de personalidad, como la alexitimia, también han sido asociados a diferentes trastornos. La alexitimia es un rasgo de personalidad derivado de observaciones clínicas que se ha definido como: a) una dificultad en identificar y describir sentimientos; b) una dificultad para distinguir entre sentimientos y sensaciones corporales; c) falta de imaginación y capacidad de fantasear, y d) una preocupación por acontecimientos externos. Esta limitada conciencia emocional y afectiva - característica central de los sujetos alexitímicos - facilitaría la amplificación de los sensaciones físicas de las emociones explicando, en gran medida, la tendencia de los sujetos alexitímicos a desarrollar trastornos por somatización y psicosomáticos, presentando, pues, una mayor vulnerabilidad al estrés. En este sentido no es de extrañar que los sujetos alexitímicos presenten con una frecuencia significativa estrategias para modular las emociones basadas en la actuación (por ejemplo, abuso de sustancias o ingesta compulsiva de comida tal como refieren Taylor y cols., 1988). Por otra parte, los trabajos de Kobasa apuntan a la existencia de un constructo de personalidad ("Hardiness") que podría actuar como factor protector de las enfermedades (Kobasa et al., 1985).

El temor al Síndrome de la Inmunodeficiencia Adquirido (SIDA) y el grado de conocimiento de la conductas de riesgo de infección al Virus de la Inmunodeficiencia Humana (VIH) son dos variables que se han mostrado capaces de producir cambios en las conductas de riesgo de adictos a drogas por vía parenteral (ADVP) de Londres y Nueva York, siendo los sujetos que manifestaban más miedo los que introducían mayores cambios en sus conductas para disminuir el riesgo de infección (DuPont, 1991).

En el caso de la infección por el VIH y del SIDA, se han propuesto diferentes estrategias de intervención psicológicas con el fin de reducir los niveles de estrés que sufren los pacientes y, por consiguiente, evitar la inmunosupresión causada por el estrés, logrando de esta manera una mejora en la calidad de vida por 
parte del paciente y un enlentecimiento en la desarrollo de la enfermedad (LaPerriere y col., 1992).

Casi todas estas variables han sido estudiadas en relación con el proceso de enfermar de SIDA o bien con diferentes variables inmunológicas. Sin embargo, no tenemos constancia de estudios que hayan analizado cómo estan estas variables psicológicas interrelacionadas entre sí antes de proceder a estudiar el conjunto de estas variables con las variables inmunológicas o relacionadas con la evolución de la enfermedad. En el presente trabajo, el objetivo es determinar cómo se agrupan estas variables psicológicas y que grado de relación hay entre ellas en sujetos ADVP. Esto nos permitiriá crear un modelo que podremos relacionar en estudios posteriores con variables inmunológicas.

\section{MÉTODO}

\section{Sujetos}

La muestra estaba formada por 107 pacientes diagnosticados de Trastorno por Dependencia de Opiáceos según criterios DSM-III-R a pesar que a la mitad del estudio se publicó el DSM-IV, se prefirió continuar con el mismo criterio para mantener la muestra lo más homogénea posible. Sesenta y siete sujetos (62.6\%) eran seropositivos al VIH y 40 (47.4\%) seronegativos. Setenta y siete eran hombres con una media de edad de 26.32 y una desviación estándar de 6.3 mientras que las 30 mujeres presentaban una media de edad de 24.45 y una desviación estándar de 4.98 . La media de los años de evolución del trastorno por dependencia de opiáceos para todos los sujetos es de 5.4 y la desviación estándar de 4.05 siguiendo todos ellos tratamiento ambulatorio. (Ver tabla 1).

\section{Instrumentos}

Los autoinformes administrados fueron los siguientes:

a) Escala de Estrés Percibido (EEP): consta de 14 items que hacen referencia a sentimientos y pensamientos experimentados relacionados con el estrés y que el sujeto debe contestar mediante respuestas tipo Likert (de 0 a 4 ) de la que se obtiene una única puntuación global de estrés percibido (Cohen y col., 1983).

b) Test de Conocimiento de Conductas de Riesgo para el contagio o transmisión del VIH (CCR): consta de 40 ítems sobre conductas de riesgo de infección o transmisión del VIH que el sujeto debe contestar mediante respuestas de Verdadero/Falso obteniéndose una puntuación global (Kelly y col., 1989).

c) Locus de Control respecto al desarrollo del SIDA (LCS): consta de 9 items con respuestas tipo Likert de 5 puntos (de "Totalmente en desacuerdo" hasta "Totalmente de acuerdo") de los que se obtienen tres subescalas; Interno, Azar y Externo (Kelly y col., 1990).

d) Escala de Temor al SIDA (ETS): consta de 38 items que hacen referencia a experiencias que pueden causar miedo o temor y que el sujeto debe responder con una respuesta tipo Likert (de 1 a 5) según el grado de temor o miedo que experimenta. Se han obtenido dos subescalas: una hace referencia al temor de desarrollar el SIDA asociado a la conducta sexual y la otra subescala hace referencia al miedo a exponerse al VIH y a otros virus (Arrindell y col., 1989).

e) Adaptación de la Escala de Ajuste Mental al Cáncer (EAM): consta de 40 items que hacen referencia a la adaptación, tanto cognitiva como conductual, a una enfermedad evaluándose la respuesta mediante respuestas tipo Likert de 4 puntos (Watson y col., 1988).

f) Escala de Alexitimia de Toronto (EAT): consta de 26 items con respuestas tipo Likert de 5 puntos (que van desde "Totalmente en desacuerdo" hasta "totalmente de acuerdo") obteniéndose una única puntuación respecto el grado en que el sujeto posee rasgos alexitímicos (Taylor y col., 1988).

g) Inventario de Estrategias de Afrontamiento respecto al SIDA (IEAS): es un inventario de 47 ítems con respuestas tipo Likert de 5 puntos (de "Nunca" a "Siempre") que evalúa respuestas cognitivas y conductuales que el sujeto realiza para afrontar la enfermedad derivándose tres escalas: Afrontamiento Activo-Cognitivo, Afrontamiento ActivoConductual y Afrontamiento de Evitación (Namir y col., 1990).

h) Cuestionario de Apoyo Social (CAS): un cuestionario de 8 ítems a los que debían contestar en una escala de 5 puntos tipo Likert el grado de deseo de ese tipo de ayuda, si está disponible, cuantas veces la ha necesitado y si ha sido de utilidad para él (Zich y Temoshok, 1990).

\section{Procedimiento}

Los autoinformes fueron administrados individualmente por un psicólogo clínico siguiendo en todos los casos el mismo orden. Asimismo, se recogieron datos sociodemográficos, datos respecto al consumo de sustancias, las conductas de riesgo realizadas en los últimos seis meses y el tiempo transcurrido desde que conoció por primera vez su seropositividad o por 
última vez su seronegatividad. Algunos ítems (3) de la escala EAM fueron modificados para que se ajustasen a la infeción por VIH o al SIDA.

\section{Análisis de Datos}

Debido a que el objetivo del estudio se centraba en analizar las relaciones que existen entre las diversas variables psicológicas que en estudios anteriores se habían mostrado relevantes se procedió a un análisis factorial de las escalas obtenidas con los distintos cuestionarios con el método de componentes principales y rotación varimax mediante el programa estadístico SPSS. Teniendo en cuenta que no existe ningún modelo teórico que abarque todas las variables psicológicas nos planteamos el realizar un análisis factorial exploratorio para determinar los factores que pueden agrupar o relacionar las variables psicológicas. Debido a que el método de extracción de factores de KaiserGuttman (eigenvalues mayores de 1) tiene tendencia a obtener un número mayor de factores de lo necesario, se optó también por extraer el número de factores a través del procedimiento del scree-test (Cattell, 1966; Loehlin, 1992) con el objetivo de conseguir el modelo más parsimonioso e interpretable posible.

\section{Resultados}

En el análisis en componentes principales con rotación Varimax de los diferentes autoinformes se obtuvo una solución de 7 factores con valores propios superiores a 1, que explicaba el $77.4 \%$ de la varianza. A pesar de que la solución aportada por los 7 factores era congruente se decidió reducir la solución a 3 factores, que explicaban el $48.8 \%$ de la varianza, siguiendo el criterio del scree-test (Cattell, 1966). La solución obtenida es la siguiente (entre paréntesis figura el porcentaje de varianza explicado por cada componente): Componente 1, Desesperanza (21.5\%); Componente 2, Racionalización y Emocionabilidad (14.6\%); Componente 3, Acción (12.7\%) (Ver Tabla 1).

\section{Discusión}

Del análisis de componentes principales se derivan diversas conclusiones de especial relevancia en el campo clínico. En el factor 1, el miedo y la angustia al desarrollo del SIDA hace aumentar la percepción del estrés y el desarrollo de conductas de evitación como estrategia de afrontamiento para disminuir la amenaza de peligro así como un cierto fatalismo y externalización del problema con muy poca capacidad para cambiar algo vivido como irremediable y experimentado con ansiedad, fatalidad y desesperanza. El factor 2 abarcaría la existencia y utilidad del apoyo social. El hecho de que las escalas de Alexitimia (TAS) y el grado de conocimiento de conductas de riesgo (CCR) puntúen negativamente en este factor sugiere que el apoyo social sería de mayor utilidad en personas que no tuviesen características alexitímicas y sin un conocimiento claro de las conductas de riesgo de infección. El hecho de que el Apoyo Social actúe en un factor ortogonal a los otros factores debe hacernos reflexionar respecto a si la utilidad de este recurso como modulador o amortiguador del estrés sólo puede darse en sujetos no alexitímicos o sin un nivel elevado de información respecto a las conductas de riesgo. El factor 3 hace referencia a todas aquellas conductas que suponen una implicación en el tratamiento, con una internalización de las estrategias cognitivo-conductuales de afrontamiento de la enfermedad y con expectativas de futuro y de resultados en función de las medidas adoptadas.

Estos factores reflejarían distintas formas de afrontamiento a la infección por $\mathrm{VIH}$ y al desarrollo del SIDA. Desde un punto de vista teórico sería de esperar poder encontrar diferencias en el proceso de enfermar (infecciones oportunistas, ingresos hospitalarios, variación en los parámetros inmunológicos, etc..) entre personas que manifiestan un tipo de afrontamiento o otro. Las personas que adoptasen un patrón de afrontamiento que puntuasen alto en el Factor 1 (Miedo y Angustia respecto al SIDA) podrían beneficiarse de técnicas cognitivas psicoeducativas y de técnicas de relajación así como de técnicas de reestructuración cognitivo-conductuales ya que la adopción de este patrón podría comprometer, a nivel teórico y considerando las aportaciones empíricas de la Psiconeuroinmunología, su sistema inmunitario y, consecuentemente, su calidad de vida.

El Factor 2 (Racionalización y Emocionabilidad) indicaría la dirección en que se centra el afrontamiento que realiza el sujeto, si centrado en los problemas o bien centrado en las emociones. Un sujeto con una alta puntuación en este factor buscaría el apoyo social como modulador de sus emociones al no presentar rasgos alexitímicos y no poseer conocimientos relevantes respecto las conductas de riesgo; por contra, un sujeto con una baja puntuación en este factor presentaría rasgos alexitímicos, conocimiento de las conductas de riesgo y no buscaría apoyo social debido a su dificultad en identificar y describir sentimientos y a su preocupación por acontecimientos externos. El entrenamiento en estrategias de afrontamiento desarrollado por Chesney y Folkman (1994) es especialmente idóneo ya que permite al individuo ajustar el tipo de afrontamiento a la situación estresante adoptando una estrategia centrada en el problema en aquellas situaciones que puedan ser cambiadas y usando una estrategia centrada en la emoción cuando existan elementos no modificables en la situación. Este tipo de entrenamiento sería beneficioso para aquellas personas que mostrasen la utilización de una 
única estrategia (Racionalización o Expresión) especialmente si se realiza en grupo con la finalidad de que aprendan a expresar verbalmente sus emociones.

Los sujetos que mantuvieran una actitud de espíritu de lucha serían los que, a nivel teórico, pondrían en menor riesgo su sistema inmunológico con lo que las estrategias de intervención deberían ser mínimas en función de las demandas y necesidades del paciente.

Los autoinformes pueden ayudar a delimitar diferentes tipologías de pacientes que requieran intervenciones terapéuticas bien diferenciadas. Sin embargo, es necesario la realización de nuevos estudios en diferentes poblaciones que permitan validar la posible existencia de distintas tipologías así como de la utilidad de las estrategias de intervención requeridas. También es imprescindible realizar estudio que permitan estudiar la relación de estos factores con variables inmunológicas.

\section{BIBLIOGRAFÍA}

ADER, R. (1981) Psychoneuroimmunology. Academic Press, San Diego.

ADER, R., FELTEN, D.L., COHEN, N. (1991). Psychoneuroimmunology, Second Edition. Academic Press, San Diego.

ARRINDELL, W.A., ROSS, M.W., BRIDGES, K.R., VAN HOUT, W., HOFMAN, A., SANDERMAN, R. (1989). Fear of AIDS: are ther replicable, invariant questionnaire dimensions?. Advances in Behaviour and Research Therapy, 11: 69-115.

BYRNES, D.M., ANTONI, M.H., GOODKIN, K., EFANTIS POTTER, J., ASTHANA, D., SIMON, T., MUNAJJ, J., IRONSON, G., FLETCHER, M.A. (1998). Stressful events, pessimism, natural killer cell citotoxicity, and citotoxic/suppressor T cells in HIV+ black women at risk for cervical cancer. Psychosomatic Medicine, 60: 714-722.

CATTELL, R.B. (1966). The scree test for the number of factors. Multivariate Behavioral Research, 1: 245-276.

CHESNEY, M.A., FOLKMAN, S. Psychological impact of HIV disease and implications for intervention. En L.S. Zegans y T.J. Coates (Eds.), Psychiatric manifestations of HIV disease (pp. 163-182), Psychiatric Clinics of North America, 17, 1, March, 1994.

COHEN, S., KAMARCK, T., MERMELSTEIN, R. (1983). A global measure of perceived stress. Journal of Health and Social Behavior, 24: 385-396.

DUPONT, E.H. (1991). Fear of AIDS among intravenous drug users in London and New York. The International Journal of the Addictions, 26: 203-212.

ENGEL, G. (1977). The need for a new medical model a challenge for biomedicine. Science, 196: 129-136.

KELLY, J.A., ST. LAWRENCE, J.S., HOOD, H.V., BRASFIELD, T.L. (1989). Behavioral intervention to reduce AIDS risk activities. Journal of Consulting and Clinical Psychology, 57: 60-67.

KELLY, J.A., ST. LAWRENCE, J.S., BRASFIELD, T.L., LEMKE, A., AMIDEI, T., ROFFMAN, R.E., HOOD, H.V., KILGORE, H., SMITH, J.E., MCNEILL JR., C. (1990). Psychological Factors that predict AIDS high-risk versus AIDS precautionary behavior. Journal of Consulting and Clinical Psychology, 58: 117-120.

KOBASA, S., MADDI, S., PUCCETTI, M., ZOLA, M. (1985). Effectiveness of hardiiness, exercise, and social support as resources against illness. Journal of Psychosomatic Research, 29: 525-533.

LAPERRIERE, A., ANTONI, M., FLETCHER, M.A., SCHNEIDERMAN, N. (1992). Exercise and Health Maintenance in HIV. En M.L. Galantino (Ed.) Clinical Assessment and Treatment of HIV. Rehabilitation of a Chronic IIIness (pp 65-76). Thorofare, New Jersey, SLACK Incorporated.

LLOR, B., GODOY, C., NIETO, J. (1993). Aspectos teóricos en conducta de enfermedad. Anales de Psiquiatría, 9, 3: $140-145$

LOEHLIN, J.C. (1992) Latent Variable Models: anintroduction to factor, path, and structural analysis. Second Edition. Lawrence Erlbaum Associates, New Jersey.

MECHANIC, D. (1962). The concept of illness behaviour. J. Chron. Dis., 15: 189-194.

MECHANIC, D. (1986). The concept of illness behaviour-culture, situation and personal predisposotion. Psychological Medicine, 16: 1-7.

MILLER, G.E., KEMENY, M.E., TAYLOR, S.E., COLE, S.W., VISSCHER, B.R. (1997). Social relationships and immune processes in HIV seropositive gay and bisexual men. Ann. Behavioral Medicine, 19:2, 139-51.

NAMIR, S., WOLCOTT, D.L., FAWZY, F.I., ALUMBAUGH, M.J. (1990). Implications of different strategies for coping with AIDS. En A. Baum y L. Temoshok (Eds.), Psychosocial Perspectives on AIDS (pp 173-190). Hillsdale, New Jersey, Lawrence Erlbaum Associates.

PILOWSKY, I. (1986). Abnormal illness behaviour. Dysnosognosia. Psychoter. Psychosom., 46, :76-84

SOLOMON, G.F., TEMOSHOK, L. (1990). A psychoneuroimmunologic perspective on AIDS research: Questions, preliminary findings and suggestions. En A. Baum y L. Temoshok (Eds.), Psychosocial Perspectives on AIDS (pp 239-2258). Hillsdale, New Jersey, Lawrence Erlbaum Associates.

TAYLOR, G.J., BAGBY, M., RYAN, D.P., PARKER, J.D.A., DOODY, K.F., KEEFE, P. (1988). Criterion Validity of the Toronto Alexithymia Scale. Psychosomatic Medicine, 50: 500-509.

WATSON, M., GREER, S., YOUNG, J., INAYAT, Q., BURGESS, C., ROBERTSON, B. (1988). Development of a questionnaire measure of adjustment to cancer: the MAC scale. Psychological Medicine, 18: 203-209.

ZICH, J., TEMOSHOCK, L. (1990). Perceptions of social support, distress, and hopelessness in men with AIDS and ARC: Clinical implications. En A. Baum y L. Temoshok (Eds.), Psychosocial Perspectives on AIDS (pp 201- 
Tabla 1: Datos Sociodemográficos de la muestra

$\mathrm{VIH}+$

$\mathrm{N}^{\circ}$ de Sujetos

Edad (Media y s.d.)

Tiempo de conocimiento de su

seropositividad (media y s.d.)

Tiempo de abstinencia (días)

Año de evolución de la adicción (media y s.d.)

Porcentaje de Relaciones sexuales con

preservativo (media y s.d.)
67 (23 mujeres) 40 (7 mujeres)

29.07 años (4.58) 28.90 años (4.75)

$60.7(9.3)$

$45.3(11.3)$

$47.5(12.2)$

$56.2(13.1)$

$7.4(4.35)$

$6.5(4.5)$

$56.80(43.05)$

$34.05(44.49)$
TOTAL MUESTRA

107

29.11 años (4.65)

$53.8(10.8)$

48.8 (11.9)

$6.78(4.43)$

$48.6(43.96)$

\section{TABLA 2: Solución factorial estandarizada}

\section{Escala}

Fatalidad (EAM)

Desesperanza (EAM)

Estrés Percibido (EEP)

Ansiedad Preocupación (EAM)

Afrontamiento de Evitación (IEAS)

Miedo a la infección (interpersonal) (ETS)

Miedo a la infección (sexual) (ETS)

Locus de Control Externo (LCS)

Apoyo Social Recibido (CAS)

Disponibilidad Apoyo Social (CAS)

Utilidad Apoyo Social (CAS)

Conocimiento Conductas de Riesgo (CCR)

Alexitimia (TAS)

Apoyo Social Deseado (CAS)

Afrontamiento Conductual (IEAS)

Locus de Control Interno (LCS)

Afrontamiento Cognitivo (IEAS)

Espíritu de Lucha (EAM)

Locus de Control al Azar (LCS)
FACTOR 1 FACTOR 2 FACTOR 3

$\begin{array}{rrr}.75528 & -.23600 & -.11455 \\ .75443 & -.14347 & -.15294 \\ .70016 & -.00583 & .12411 \\ .63159 & -.25103 & .32994 \\ .63151 & .09410 & .04731 \\ .59807 & .22132 & .04945 \\ .58223 & .33175 & .11996 \\ . \mathbf{4 3 1 4 7} & .03445 & .07858 \\ & & \\ .12733 & .84979 & -.00376 \\ .08652 & . \mathbf{7 8 4 5 4} & .01344 \\ -.09316 & . \mathbf{6 2 0 4 8} & -.10078 \\ -.35179 & -.50881 & .07554 \\ .33017 & -.46553 & -.27472 \\ -.04597 & .40943 & .07204 \\ & & \\ -.08449 & .06427 & . \mathbf{8 6 8 9 3} \\ .19688 & .20115 & . \mathbf{7 3 6 9 0} \\ .43897 & .01173 & \mathbf{. 6 2 9 5 3} \\ .12216 & -.03390 & \mathbf{. 6 1 3 5 1} \\ .30871 & .17859 & -.48408\end{array}$

227). Hillsdale, New Jersey, Lawrence Erlbaum Associates. 